\title{
An empirical method to estimate the LMC distance using B-stars in eclipsing binary systems
}

\author{
M. Salaris ${ }^{1,2}$ and M. A. T. Groenewegen ${ }^{3,4}$ \\ 1 Astrophysics Research Institute, Liverpool John Moores University, Twelve Quays House, Egerton Wharf, \\ Birkenhead CH41 1LD, UK \\ 2 Max-Planck-Institut für Astrophysik, Karl-Schwarzschild-Straße 1, 85748 Garching, Germany \\ 3 Instituut voor Sterrenkunde, PACS-ICC, Celestijnenlaan 200B, 3001 Heverlee, Belgium \\ 4 European Southern Observatory, EIS-team, Karl-Schwarzschild-Straße 2, 85740 Garching, Germany
}

Received 22 August 2001 / Accepted 29 October 2001

\begin{abstract}
We present a new method to determine the distance to B-stars in eclipsing binary systems. The method is completely empirical, and it is based on the existence of a very tight linear relationship between the $V$-band "zero magnitude angular diameter" and the Strömgren colour index $c_{1}$ for B-stars; we have empirically calibrated this relationship using local single B-stars with accurate angular diameters, and B-stars in eclipsing binaries with precise radii and parallax determinations. By studying the differential behaviour of this relationship as predicted by theoretical stellar evolution models, we find that it is independent of the stellar metallicities for a range of $[\mathrm{Fe} / \mathrm{H}]$ values between the solar one and that of young stars in the Magellanic Clouds. The method, which also provides the value of the reddening to the system, is discussed in detail, together with a thorough estimate of the associated errors. We conclude that accurate Strömgren photometry obtainable with $1.5 \mathrm{~m}$-class telescopes of the LMC eclipsing binaries HV 2274 and HV 982 will allow to obtain an empirical LMC distance with an accuracy of the order of $0.13 \mathrm{mag}$.
\end{abstract}

Key words. binaries: eclipsing - stars: distances - stars: individual: HD 24909, HD 161783, HD 218066, HV 982, HV 2274 - stars: fundamental parameters - Magellanic Clouds - distance scale

\section{Introduction}

The distance to the large Magellanic Cloud is the cornerstone of the extragalactic distance scale, since the zero point of both the Cepheids and Type Ia supernovae distances is tied to the LMC distance. Disappointingly enough, existing determinations of this fundamental quantity span a wide range, comprising both "short" $\left((m-M)_{0} \leq 18.30\right.$ - e.g. Udalski 2000) and "long" $\left((m-M)_{0} \geq 18.50-\right.$ e.g. Feast \& Catchpole 1997) values.

In the last few years well-detached main sequence B type eclipsing binary (EB) systems have been proposed as virtually ideal standard candles, and employed by Guinan et al. (1998) and Fitzpatrick et al. (2001 - F01) to derive the distance to two EB systems in the LMC (HV 2274 and HV 982). From an observational point of view, what is required is the monitoring of the light curve (preferably in several filters) and of the radial velocity. Analysing this data gives the orbital elements, the masses and radii of both components. In addition to that, UV/optical spectra are necessary; once obtained,

Send offprint requests to: M. Salaris,

e-mail: ms@astro.livjm.ac.uk a multi-parametric fit of theoretical spectra to the observed spectrum and the broadband data is performed, in order to simultaneously derive the effective temperature of both components, reddening, metallicity, microturbulent velocity, the distance and 5 parameters describing the UV/optical extinction curve. When applying this method to HV2274, Guinan et al. (1998) and F01 found, respectively, $(m-M)_{0}=18.30$ and $(m-M)_{0}=18.36$, with an error on the individual determinations of the order of 0.10 mag; F01 obtained $(m-M)_{0}=18.50 \pm 0.06$ for HV982. As shown by Groenewegen \& Salaris (2001 GS01) for the case of HV 2274, this method heavily relies on the absolute value of the fluxes predicted by the theory, which, given the current uncertainty of stellar atmospheres modelling (see GS01 for more details), can introduce large systematic errors. Moreover, GS01 have shown that the resulting distance is also dependent on what broadband colours are included in the fitting procedure, so that, even if a formal error of the order of $0.10 \mathrm{mag}$ can be derived from the fitting procedure, the real uncertainty is probably much larger. 
An alternative method to use EB systems as distance indicators is based on empirical relationships linking colours to surface brightness (see, e.g., Barnes \& Evans 1976; Lacy 1977; Di Benedetto 1998 and references therein). Once the colours of the individual components of the system are known from the light curve analysis (e.g. Lacy 1977), these relationships provide the apparent radii; since the true radii are also known from the analysis of the radial velocity curve, the distance to the system is derived straightforwardly. This method has been applied recently by Thompson et al. (2001) to one EB system in the turn-off region of the Colour-Magnitude-Diagram of the globular cluster $\omega$ Centauri. They made use of surface brightness (in the $V$-band) versus colour relationships in $(V-K),(V-H),(V-J)$, calibrated on the stellar sample by Di Benedetto (1998), for A, F, G, K dwarfs and giants. This method is very much appealing, since it avoids the use of the still uncertain model atmospheres. One drawback is that it is necessary to know in advance the reddening of the system, which is not an easy task if one wants to apply this technique to LMC objects. Moreover a calibration for B-stars - like the stars in the LMC EB systems previously discussed - does not exist yet.

In this paper we propose a variant of this surface brightness technique using Strömgren colours, suited to determine the distance of B-stars in EB systems. This method turns out to be largely independent of the metallicity of the components and, moreover, working with the Strömgren filters one can easily derive the reddening of B-stars from the $c_{1}-(b-y)$ diagram (see, e.g., Larsen et al. 2000 - LCS00).

In Sect. 2 we discuss the theoretical background of the method, while in Sect. 3 we present our calibration and use for B-stars. Section 4 discusses the errors associated with the method through an application to some Galactic EB systems and the LMC system HV 982. Conclusions follow in Sect. 5.

\section{Theoretical background}

The surface brightness $(S)$ of a star is classically defined as:

$S_{\lambda}=m_{\lambda}+5 \log \phi$

where $\phi$ is the angular diameter in milli-arcseconds, and $m_{\lambda}$ represents the apparent magnitude in a given passband. $S_{\lambda}$ is determined empirically by measuring the stellar angular diameters and brightness of non-variable stars.

A slightly alternative concept, but essentially equivalent, is used by van Belle (1999) to define the angular diameter a star would have if its magnitude (in a given passband) were zero (zero magnitude angular diameter):

$\phi_{\left(m_{\lambda}=0\right)}=\phi 10^{m_{\lambda} / 5}$

where $\phi$ is again the angular diameter.

In either case, $S_{\lambda}$ and $\phi_{\left(m_{\lambda}=0\right)}$ are empirically calibrated against a colour $\left(m_{2}-m_{3}\right)$ on a sample of local

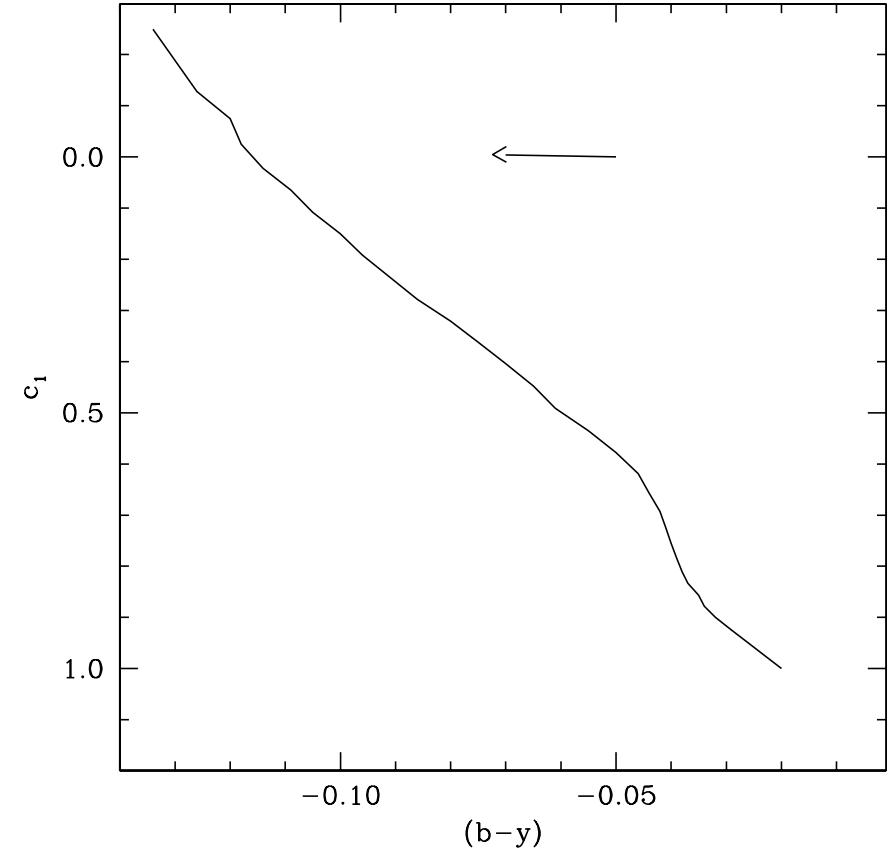

Fig. 1. Standard sequence for B-stars. The arrow shows the direction of the reddening vector.

stars. In the case of well-detached EBs, magnitudes and colours for the individual components are derived using information from the light-curve analysis, and dereddened. The observed colours and magnitudes, together with the empirical relationships previously discussed, provide the angular diameters (in mas) of the two components. From the angular diameter and the absolute radius $(R)$ obtained from the analysis of the radial velocity curve, one obtains easily the distance to the two components according to the relationship:

$d(\mathrm{pc})=1.337 \times 10^{-5} R(\mathrm{~km}) / \phi(\mathrm{mas})$.

It is obvious that in order to apply this method one needs to know the radius of the stars, therefore it can be reliably applied only to EB systems (but see also Allende Prieto 2001). In principle one can use any magnitude and colour in conjunction with Eqs. (1) or (2).

\section{Calibration for B-stars with Strömgren photometry}

When trying to apply surface brightness techniques one must face the problem of reddening. The reddening in, e.g., the LMC is strongly variable on very short spatial scales (see, e.g., Romaniello et al. 2000, LCS00), and the ideal way to proceed is to determine it on a star-by-star basis. Strömgren uvby photometry provides the tool to do this, at least for B-stars, by employing the $c_{1}-(b-y)$ diagram (we briefly recall that $c_{1}=(u-v)-(v-b)$ ). As discussed by Larsen et al. (2000), in this plane luminosity class IV and V B-stars are located on a standard sequence (see Table 1) which is largely independent of the star metallicity (LCS00). In Fig. 1 we show the standard sequence by Perry et al. (1987), and the direction 
Table 1. Standard sequence for B-stars (from Perry et al. 1987).

\begin{tabular}{cccc}
\hline$(b-y)$ & $c_{1}$ & $(b-y)$ & $c_{1}$ \\
\hline-0.134 & -0.250 & -0.050 & 0.578 \\
-0.126 & -0.128 & -0.046 & 0.619 \\
-0.120 & -0.075 & -0.044 & 0.656 \\
-0.118 & -0.025 & -0.042 & 0.693 \\
-0.114 & 0.022 & -0.041 & 0.724 \\
-0.109 & 0.065 & -0.040 & 0.755 \\
-0.105 & 0.108 & -0.039 & 0.785 \\
-0.100 & 0.150 & -0.038 & 0.811 \\
-0.096 & 0.192 & -0.037 & 0.833 \\
-0.091 & 0.235 & -0.035 & 0.856 \\
-0.086 & 0.278 & -0.034 & 0.878 \\
-0.080 & 0.321 & -0.032 & 0.900 \\
-0.075 & 0.362 & -0.029 & 0.925 \\
-0.070 & 0.404 & -0.026 & 0.950 \\
-0.065 & 0.448 & -0.023 & 0.975 \\
-0.061 & 0.491 & -0.020 & 1.000 \\
-0.055 & 0.535 & & \\
\hline
\end{tabular}

of the reddening vector, which is nearly horizontal, since $\left(c_{1}\right)_{0}=c_{1}-0.20 E(b-y)$. To first order, the reddening $E(b-y)$ can be estimated as simply being the difference between the observed $(b-y)$ and the intrinsic value $(b-y)_{0}$ corresponding to the observed $c_{1}$. We recall that $E(B-V)=1.4 E(b-y)$.

To confirm the independence of this standard sequence on metallicity, we show in Fig. 2 the $c_{1}-(b-y)$ relationship for B-stars between the zero age main sequence and the turn off (luminosity classes IV and V) with ages between 10 and $50 \mathrm{Myr}$ (the precise age range is not relevant), from the theoretical models by Bertelli et al. (1994), transformed to the Strömgren filters by means of the Kurucz (1992) colour transformations. In the $[\mathrm{Fe} / \mathrm{H}]$ range displayed $-[\mathrm{Fe} / \mathrm{H}]$ between 0.0 and -0.7 , which covers the metallicity range of B-stars in the Milky Way, LMC and SMC (see, e.g., the discussion in GC01) - the standard sequence of B-stars is practically unaffected by the stellar chemical composition. The maximum difference is less than $0.01 \mathrm{mag}$ in $(b-y)$ at a given value of $c_{1}$ between the $[\mathrm{Fe} / \mathrm{H}]=-0.7$ and 0.0 sequence.

Therefore, as a first step, after the radial velocity and light curve analysis (from Strömgren filter observations) of a given EB system with B main sequence star components, one can derive the reddening of the two objects by simply using the $c_{1}-(b-y)$ diagram and the sequence provided in Table 1.

As a second step, one needs a relationship between the surface brightness or zero magnitude angular diameter, and a de-reddened colour. The following precepts have guided our choice:

- the relationship should be tight and mathematically simple;

- there has to be a sufficient number of local calibrators;

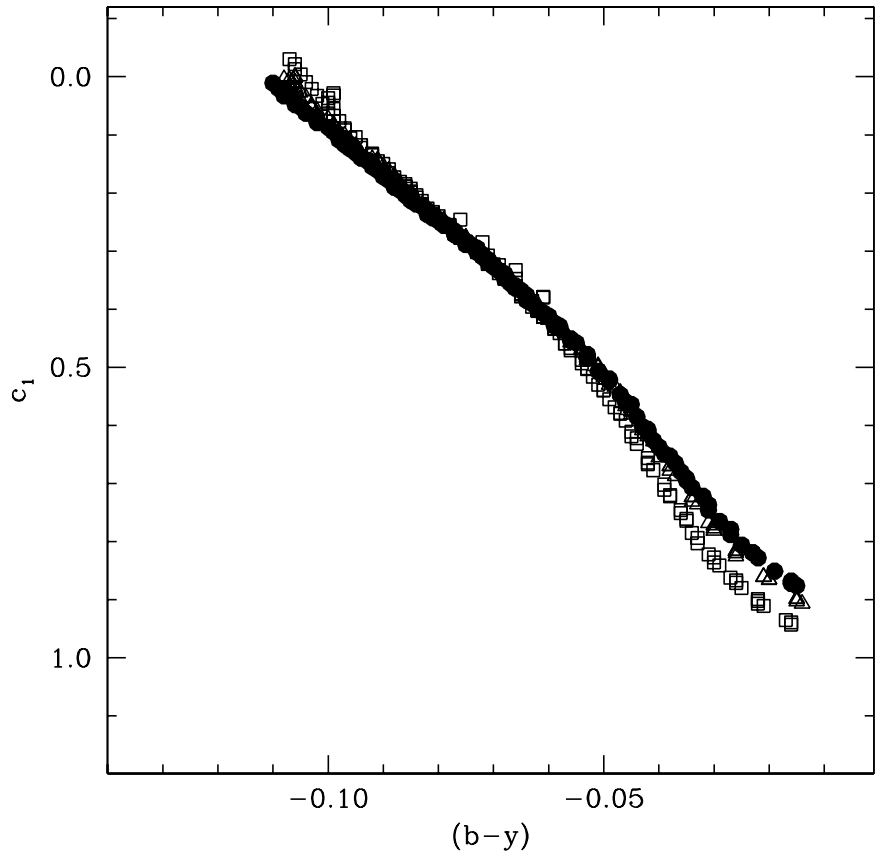

Fig. 2. Theoretical sequences for main sequence B-stars with ages between 10 and $50 \mathrm{Myr}$ and $[\mathrm{Fe} / \mathrm{H}]=-0.7$ (filled circles), -0.4 (open triangles) and 0.0 (open squares).

- it has to be dependent as weakly as possible on photometric errors, reddening uncertainties, and differences in the chemical composition between calibrating and observed objects.

We found that a relationship fulfilling all these constraints does exist for B-stars, and links $\phi_{(V=0)}$ with $c_{1}$. In Table 2 we provide the values of $\phi_{(V=0)}, c_{1}$ and $(b-y)$ (reddening corrected) for the 9 calibrating B-stars we found in the literature; they cover fairly well the entire range of B main sequence stars. Four of these objects are in 2 EB systems with well determined Hipparcos parallaxes $-\sigma_{\pi} / \pi<12 \%$ - (photometry and radii are taken from Jordi et al. 1997; Ribas et al. 1998) and radii with an error of less than $2 \%$; their absolute radii have been therefore transformed into angular diameters as if they were located at a distance of $10 \mathrm{pc}$, which have been then used, together with the absolute $V$ magnitudes, to compute $\phi_{(V=0)}{ }^{1}$. The remaining stars have direct angular diameter determinations accurate to better than 9\% from Code et al. (1976), and photometry obtained from the Mermilliod et al. (1997) catalogue. Errors on the photometric data come from Jordi et al. (1997) for the EB systems, while we have

\footnotetext{
1 Data for other EB systems were available with the appropriate photometric and radius errors, but the uncertainty on their parallaxes was higher, thus causing a very large uncertainty on their $\phi_{(V=0)}$ values. Since the 2 systems we choose have been selected on the base of their parallax error, we applied the Lutz-Kelker correction to the distances obtained from their parallax, following Smith (1987), and assuming a uniform stellar distribution. The corrections applied to the absolute $V$ magnitudes of the EB systems are -0.046 and -0.149 mag for, respectively, HD 6882 and HD 135876.
} 
Table 2. Calibrating stars for the $\phi_{(V=0)}-c_{1}$ relationship. The photometric data $\left(c_{1},(b-y)\right.$ and apparent magnitudes $\left.V\right)$ have been de-reddened (if needed) using the standard sequence in Table 1.

\begin{tabular}{cccccl}
\hline$\phi_{(V=0)}$ & $c_{1}$ & $(b-y)$ & $V$ & $E(b-y)$ & name \\
\hline $1.53 \pm 0.09$ & $0.111 \pm 0.020$ & $-0.091 \pm 0.020$ & $1.64 \pm 0.02$ & 0.00 & HD 35468 \\
$2.24 \pm 0.20$ & $0.490 \pm 0.030$ & $-0.070 \pm 0.020$ & $4.30 \pm 0.01$ & 0.00 & HD 6882A \\
$2.74 \pm 0.24$ & $0.770 \pm 0.030$ & $-0.010 \pm 0.020$ & $5.68 \pm 0.01$ & 0.00 & HD 6882B \\
$2.55 \pm 0.12$ & $0.712 \pm 0.020$ & $-0.041 \pm 0.020$ & $1.35 \pm 0.02$ & 0.00 & HD 87901 \\
$2.05 \pm 0.28$ & $0.447 \pm 0.012$ & $-0.065 \pm 0.007$ & $5.83 \pm 0.009$ & 0.01 & HD 135876A \\
$2.61 \pm 0.35$ & $0.807 \pm 0.030$ & $-0.038 \pm 0.019$ & $7.07 \pm 0.014$ & 0.01 & HD 135876B \\
$1.10 \pm 0.12$ & $-0.037 \pm 0.020$ & $-0.119 \pm 0.020$ & $1.88 \pm 0.02$ & 0.10 & HD 143275 \\
$1.96 \pm 0.13$ & $0.271 \pm 0.020$ & $-0.092 \pm 0.020$ & $1.94 \pm 0.02$ & 0.00 & HD 193924 \\
$2.27 \pm 0.16$ & $0.567 \pm 0.020$ & $-0.058 \pm 0.020$ & $1.74 \pm 0.02$ & 0.00 & HD 209952 \\
\hline
\end{tabular}

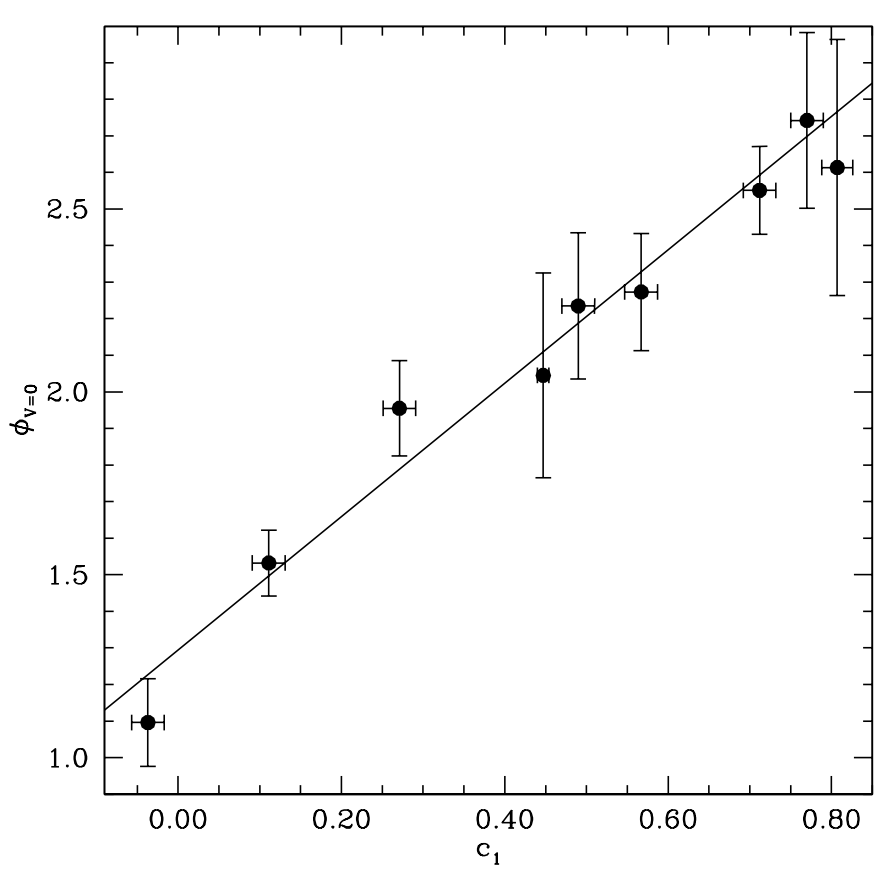

Fig. 3. Calibration of the $\phi_{(V=0)}-c_{1}$ relationship. Points are the empirical data, while the solid line shows the best fit to the data (Eq. (4)).

adopted errors of $0.02 \mathrm{mag}$ for the data obtained from the Mermilliod et al. (1997) catalogue.

In Fig. 3 we show the calibration of the empirical $\phi_{(V=0)}-c_{1}$ relationship using the data in Table 2 , which is given by

$\phi_{(V=0)}=1.824( \pm 0.180) c_{1}+1.294( \pm 0.078)$

with a linear correlation coefficient $r=0.99$ (corresponding to a probability of $\sim 1.0 \mathrm{e}-6$ that the points lie on this relationship only by chance).

At present there are no calibrations of the relationship between zero magnitude angular diameter (or surface brightness) and colours, spanning the entire main sequence B star range, other than the one we obtained. Since there are essentially no infrared colours available for our calibrating objects, neither the Di Benedetto (1998), nor the van Belle (1999) calibrations cover this range. It would be very interesting, when data will be available in the future, to compare our result with equivalent calibrations using, e.g., $(V-K)$.

An important issue is the dependence of Eq. (4) on the stellar metal content, since the calibrators are local stars of presumably solar-like metallicity, while the B-stars in the LMC are more metal poor. The only way to assess this dependence, due to the lack of appropriate data for more metal poor stars, is - as in the case of the $c_{1}-(b-y)$ standard sequence - to use the differential (not the absolute quantities) behaviour of theoretical models. Figure 4 shows in the $\phi_{(V=0)}-c_{1}$ plane the same models displayed in Fig. 2.

For B-stars of luminosity class IV and $\mathrm{V}$ one expects that the $\phi_{(V=0)}-c_{1}$ relationship is negligibly affected by the metallicity for $[\mathrm{Fe} / \mathrm{H}]$ between 0.0 and -0.7 . In the case of $[\mathrm{Fe} / \mathrm{H}]=-0.7$ (typical metallicity of SMC young stars) the difference with respect to the solar case is small for late type B-stars (higher values of $c_{1}$ ), causing a systematic underestimate of the distance (when using the local calibration) of the order of only $0.05 \mathrm{mag}$, and completely vanishing for earlier types. It is therefore possible to apply Eq. (4) with confidence also to stars in the Magellanic Clouds.

Summarizing, from the Johnson $V$ and Strömgren uvby light curves of B-stars in well-detached EB systems, one can derive the individual $V$ magnitude and Strömgren colour of the single components. Using the standard relationship in Table 1, the reddening of the system can be easily estimated. Once the de-reddened $c_{1}$ values of the 2 components are at hand, one can employ Eq. (4) to derive the corresponding zero magnitude angular diameters in $V$, which can be transformed to true angular diameters using Eq. (2). At this stage, since the angular diameters and the physical radii (derived from the analysis of the radial velocity curve) are known, one can finally use Eq. (3) to obtain the distance of the individual stars. As a check for the consistency of the results, one has to ensure that the distances of the two components are in agreement within the errors, or, equivalently, that the ratio of 
Table 3. Data for the Galactic systems analyzed (see text for details).

\begin{tabular}{lcrccccc}
\hline name & $V$ & $(b-y)$ & $c_{1}$ & radius & $E(b-y)$ & $(m-M)_{0}$ & $(m-M)_{0 . \text { Hip }}$ \\
\hline HD 24909A & $7.866 \pm 0.014$ & $0.056 \pm 0.004$ & $0.635 \pm 0.011$ & $2.446 \pm 0.026 R_{\odot}$ & $0.102 \pm 0.010$ & $7.29 \pm 0.12$ & $7.62 \pm 0.78$ \\
HD 161783A & $6.177 \pm 0.010$ & $-0.038 \pm 0.005$ & $0.249 \pm 0.010$ & $4.432 \pm 0.084 R_{\odot}$ & $0.053 \pm 0.010$ & $7.83 \pm 0.12$ & $7.01 \pm 0.52$ \\
HD 218066A & $8.35 \pm 0.04$ & $0.333 \pm 0.010$ & $0.037 \pm 0.015$ & $5.685 \pm 0.130 R_{\odot}$ & $0.455 \pm 0.010$ & $9.60 \pm 0.16$ & $9.30 \pm 1.53$ \\
HD 218066B & $8.60 \pm 0.04$ & $0.339 \pm 0.010$ & $0.045 \pm 0.015$ & $5.177 \pm 0.129 R_{\odot}$ & $0.455 \pm 0.010$ & $9.62 \pm 0.16$ & $9.30 \pm 1.53$ \\
\hline
\end{tabular}

Table 4. Data for HV 982.

\begin{tabular}{lcccccc}
\hline name & $V$ & $(b-y)$ & $c_{1}$ & radius & $E(b-y)$ & $(m-M)_{0}$ \\
\hline HV 982 A & $15.26 \pm 0.02$ & $0.008 \pm 0.041$ & $0.062 \pm 0.045$ & $8.02 \pm 0.23 R_{\odot}$ & $0.120 \pm 0.045$ & $18.43 \pm 0.24$ \\
HV 982 B & $15.48 \pm 0.02$ & $0.010 \pm 0.041$ & $0.076 \pm 0.045$ & $7.35 \pm 0.21 R_{\odot}$ & $0.120 \pm 0.045$ & $18.42 \pm 0.24$ \\
\hline
\end{tabular}

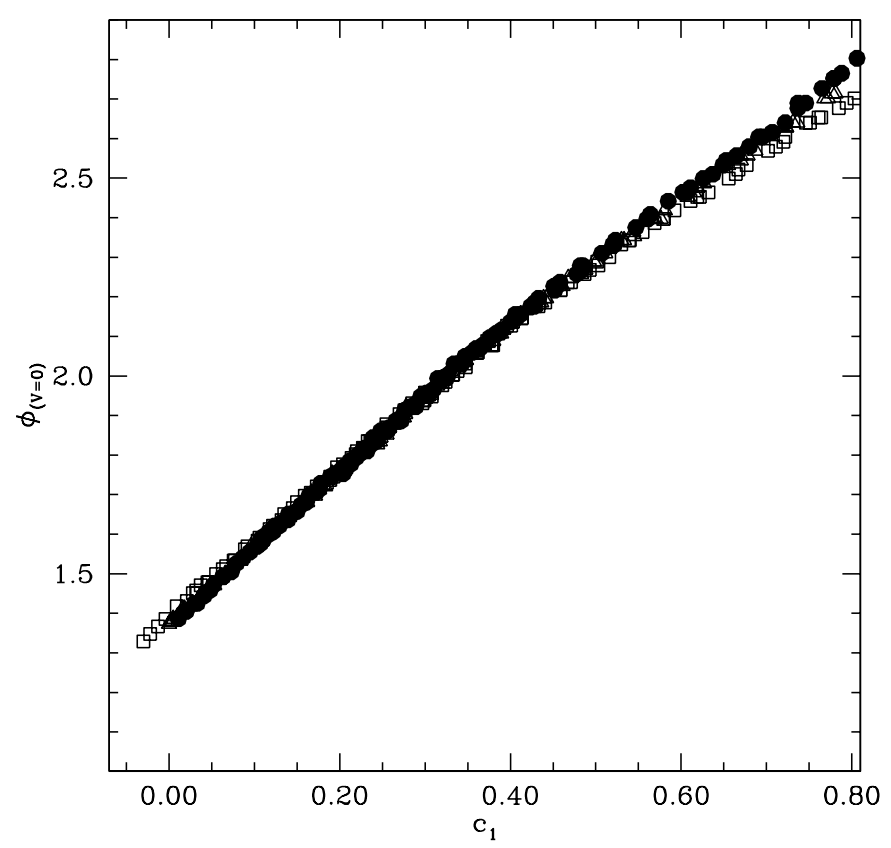

Fig. 4. Theoretical $\phi_{(V=0)}-c_{1}$ relationships for $[\mathrm{Fe} / \mathrm{H}]=-0.7$ (filled circles), -0.4 (open triangles) and 0.0 (open squares).

the derived angular diameters agrees with the observed radii ratio.

\section{Error estimate and constraints on LMC observations}

In this section we will apply the previously outlined method to some B-stars in Galactic EB systems, in order to estimate the associated errors. We have considered 3 EB systems spanning the entire spectral range of $B$ main sequence stars; the data about these systems are displayed in Table 3 . Observed magnitudes and colours (Cols. 2, 3 and 4) come from the compilation by Jordi et al. (1997), with the exception of the error bars on the Strömgren data for HD 161783, which we have computed from the original paper by Clausen (1996); radii (Col. 5) come from the compilation by Andersen (1991), while Cols. 6 and 7 show reddenings and distance moduli obtained with our technique. Finally, Col. 8 displays the distance according to the Hipparcos parallax. For HD 24909 and HD 161783 we considered just one component, since for the former system the secondary star is an A-star, while for the latter one the secondary star is variable. All 3 systems have parallaxes with large error bars; otherwise, we would have included them in our empirical calibration of Eq. (4). We want also to mention the fact that the $c_{1}$ values for both components of HD 218066, once dereddened, are slightly outside the range of validity of our calibration (by less than $0.02 \mathrm{mag}$ ). The stars in Table 3 are ordered according to their spectral type; HD 24909A is a late type B-star, while the components of the HD 218066 system are both early type B-stars. Notice the good agreement between the distances derived for the components of this system.

The errors on the distances have been derived by adding in quadrature the contributions of the errors on $V$, $(b-y), c_{1}$, the individual radii and the coefficients of Eq. (4). The largest component of the total error comes from the errors on the coefficients of Eq. (4); they alone provide an uncertainty of about $0.10-0.12 \mathrm{mag}$ on the final error on the distance modulus. Errors on the photometry and on the radii are of much less importance, as long as they are within $\sim 0.02 \mathrm{mag}$ and $3 \%$, respectively.

To summarize the results of these error estimates, our empirical method to derive distances to individual B-stars in EB systems provides distance moduli with errors of the order of $0.12-0.16 \mathrm{mag}$, when applied to systems with photometry and radius determinations precise at the level discussed before.

In case of the LMC, HV 2274 and HV 982 are EB systems with early type B-stars, for which radius determinations and $V$ photometry have a high enough precision (errors of the order of $3 \%$ on the radii and $0.02 \mathrm{mag}$ on $V$ ) to make the error on the distance dominated only by the dispersion associated to Eq. (4). Unfortunately, Strömgren data for HV 2274 are not available in the literature, while 
for HV982 the Strömgren photometry by Pritchard et al. (1998 - P98) has large errors of the order of 0.04-0.05 mag in $(b-y)$ and $c_{1}$. Nevertheless, as an exercise, we derive the distance to HV 982 following our method. In Table 4 we present the observational data for this system (Strömgren data are from P98, $V$-band data and radii are from F01) and the derived reddening and distance modulus of both components.

The derived reddening is in agreement with the estimate for B-stars in a field around HV 982 derived by LCS00 using the $(b-y)$ and $c_{1}$ indices; they find an average $E(b-y)=0.10$ with values ranging between $\sim 0.04$ and $\sim 0.25 \mathrm{mag}$. FP01 derive a reddening of $E(B-V)=0.086 \pm 0.005$ from their multidimensional spectral fitting method, consistent with the value derived here. The distances we obtain, $(m-M)_{0}=18.43 \pm 0.24$ and $(m-M)_{0}=18.42 \pm 0.24$, do not put very strong constraints on the LMC distance, given the large error bar. However, it is feasible to get Strömgren photometry accurate to $0.02-0.03 \mathrm{mag}$ in $(b-y)$ and $c_{1}$ with only 1.5 m-class telescopes (see, e.g., the data by LCS00); already with these errors one could get the distance to HV 982, for example, with an accuracy of 0.17-0.18 mag, equivalent to a parallax error of about $8 \%$. Since both HV 982 and HV 2274 lie near the LMC center (van der Marel \& Cioni 2001), if Strömgren data of the same precision were available also for HV 2274, it would be possible to combine both distances and obtain an empirical LMC distance accurate to $0.13 \mathrm{mag}$.

\section{Conclusions}

We have presented a new technique to estimate reddening and distance moduli to B-stars in EB systems. It is based on the observation of the Vuvby light curve and the consequent determination of $V, c_{1}$ and $(b-y)$ magnitudes for the individual components of the binary. A comparison of the stars' positions on the $c_{1}-(b-y)$ plane with respect to the local sequence of Table 1 permits to derive the reddening to the system. After de-reddening the individual $V$ and $c_{1}$ values, the use of an empirically calibrated $\phi_{(V=0)}-c_{1}$ relationship (Eq. (4)) allows one to derive the apparent diameter of the objects; this apparent diameter, coupled with the knowledge of the physical radius from the radial velocity analysis, provides straightforwardly the distance to the system (Eq. (3)). These two empirical relationships used to derive reddening and distance are expected to be basically independent of the stellar metallicities in the $[\mathrm{Fe} / \mathrm{H}]$ range covered by young stars in the solar neighborood, LMC and SMC. Photometric errors within $\sim 0.02 \mathrm{mag}$ and radius uncertainties up to $3 \%$ allow to determine distances to individual systems with an error bar of $0.12-0.17 \mathrm{mag}$. If this photometric accuracy is obtained for the photometry of the two LMC systems HV 2274 and HV 982 - which have both precise radius determinations and lie close to the LMC center - one can derive an empirical estimate of the LMC distance with an accuracy of the order of $0.13 \mathrm{mag}$.

Acknowledgements. M.S. wishes to thank the Max-PlanckInstitut für Astrophysik for the kind hospitality during the completion of this work. We thank Phil James for comments on a preliminary version of the manuscript. This research has made use of the SIMBAD database, operated at CDS, Strasbourg, France.

\section{References}

Allende Prieto, C. 2001, ApJ, 547, 200

Andersen, J. 1991, A\&AR, 3, 91

Barnes, T. G., \& Evans, D. S. 1976, MNRAS, 174, 489

Bertelli, G., Bressan, A., Chiosi, C., Fagotto, F., \& Nasi, E. 1994, A\&AS, 106, 275

Clausen, J. V. 1996, A\&A, 308, 151

Code, A. D., Davis, J., Bless, R. C., \& Hanbury Brown, R. 1976, ApJ, 203, 417

Di Benedetto, G. P. 1998, A\&A, 339, 858

Feast, M. W., \& Catchpole, R. M. 1997, MNRAS, 286, L1

Fitzpatrick, E. L., Ribas, I., Guinan, E. F., DeWarf, L. E., \& Maloney, F. P. 2001, ApJ [astro-ph/0010526] (FP01)

Groenewegen, M. A. T., \& Salaris, M. 2001, A\&A, 366, 752 (GS01)

Guinan, E. F., Fitzpatrick, E. L., DeWarf, L. E., et al. 1998, ApJ, 509, L21

Jordi, C., Ribas, I., Torra, J., \& Gimenez, A. 1997, A\&A, 326, 1044

Kurucz, R. 1992, IAU Symp., 149, 225

Lacy, C. H. 1977, ApJ, 213, 458

Larsen, S. S., Clausen, J. V., \& Storm, J. 2000, A\&A, 364, 455 (LCS00)

Mermilliod, J.-C., Mermilliod, M., \& Hauck, B. 1997, A\&AS, 124,349

Perry, C. L., Olsen, E. H., \& Crawford, D. L. 1987, PASP, 99, 1184

Pritchard, J. D., Tobin, W., Clark, M., \& Guinan, E. F. 1998, MNRAS, 299, 1087

Ribas, I., Gimenez, A., Torra, J., Jordi, C., \& Oblak, E. 1998, A\&A, 330, 600

Romaniello, M., Salaris, M., Cassisi, S., \& Panagia, N. 2000, ApJ, 530, 738

Smith, H. 1987, A\&A, 188, 233

Udalski, A. 2000, ApJ, 531, L25

Thompson, I. B., Kaluzny, J., Pych, W., et al. 2001, ApJ, 121, 3089

Van Belle, G. T. 1999, PASP, 111, 1515

van der Marel, R., \& Cioni, M.-R. L. 2001, AJ, 122, 1807 